\title{
The Translation of Sci-Tech English Paper Abstracts from the Perspective of Eco-Translatology
}

\author{
Yongping Zhang \\ School of Foreign Languages \\ Shaoguan University \\ Shaoguan, People’s Republic of China
}

\author{
Zaibo Li \\ School of Civil Engineering \\ Shaoguan University \\ Shaoguan, People’s Republic of China
}

\begin{abstract}
The translation of scientific paper abstracts is very important for international academic communication. Based on the eco-translatology and current existing problems in Chinese to English translation of scientific paper abstracts, this paper analyzed the translations of sci-tech abstracts in current national core journals and made the research on the aspect of multi-level reconstruction to improve the Chinese to English translation of scientific paper abstracts on the perspectives of information structure, logic cohesion, use of words and aesthetics, etc.
\end{abstract}

Keywords-Abstract translation; Eco-translatology; Multidimensional reconstruction

\section{INTRODUCTION}

The abstract is an important part of academic paper. With global development, good abstract translation gains more and more attention, because it is beneficial to promote knowledge dissemination and academic communication and cooperation. Scientific and technical workers and translators pay more and more attention to the importance of Chinese to English translation of scientific paper abstracts in our country, but for the scholars of science and engineering, the sophisticated translation of their abstracts is a tough task.

\section{THE RESEARCH SitUATION OF CHINESE TO ENGLISH TRANSLATION OF SCIENTIFIC PAPER ABSTRACTS}

The domestic research on the translation of abstracts started from the 1980s, and at the beginning of the 1980s, there were scholars who quested for the writing methods of abstracts [1]. From the year 1986 when the first relevant literature was published in Chinese Science and Technology Translators Journal, there were more and more researches on scientific paper abstracts, and after the year 2000, the researches on them increased in quantity. And among them, the researches on medical journals were the most, and the analysis on editors' demands and the error statistics of abstract translation from the perspective of the periodical editors was in the second place. Although the periodical editors were equipped with rich professional discipline knowledge, their English writing and translating competences were varied, so they can't make an inquiry on the deeper reason for the errors.

In recent years the scholars make diversified research on Chinese to English translation of scientific paper abstracts, such as vocabulary, grammatical features, syntax, discourse, etc. [2]. For example, Yao Jun analyzed generic structure and the grasp of appropriate linguistic performance for Chinese students' writing abstracts in English on discourse and linguistic form; Teng Zhen-ru and Tan Wan-cheng made the research and analysis on the tense and voice of English academic abstracts; Guo Jian-shun discussed the writing norms from the point of national standard, and researched and summarized the errors and reasons of English punctuation marks in Chinese sci-tech periodicals; in addition, there were some researches on introducing the successful experience of abstract translation [3]. The theoretical foundations that scholars and translators used on the research of abstract translation also become more and more abundant, such as the functionalist translation theory, skopostheorie, relevance theory, subjectivity of translator, textual function, etc.

\section{THE ENLIGHTENMENT OF ECO-TRANSLATOLOGY FOR ABSTRACT TRANSLATION OF SCIENTIFIC PAPER}

In China, Eco-translatology started from 2001, and was in full swing in 2009. In Translation as Adaptation and Selection, the notion of Eco-translatology was put forward formally by Hu Gengshen from Tsinghua University in 2004. This theory makes Darwin's biological evolutionism a philosophical basis and based on the basic theory of adaptive selection in evolutionism the theory of translation as adaption and selection were built first and then it developed into the current Ecotranslatology [4]. The notion of translation as adaption and selection is: translation is adaption and selection, and the best translation is the translators' cumulative effect of multidimensional adaption and adaptive selection for translational eco-environment. For translators, the fittest survives and develops; for the translation, the fittest survive and comes into effect. In short, the process of translators' translation can be regarded as the translators' adaption to the translational ecoenvironment and translators' selection. The research of translation itself is the focus of Eco-translatology and the interrelation between translators and translational ecoenvironment is its research subject, and it means the translation is explained from the perspective of translator and the translator's subjective initiative is emphasized, and thus the translation concept-translator is the center was put forward explicitly [5]. The change of translation environment makes translators suit the translational eco-environment and meanwhile, the translators' personal internal factors determine the translators' self-selections, so translators have a dual identity in Eco-translatology.

This research was financially supported by the Foundation of Shaoguan University (SY2018SK08). 
As the combining product of ecology and translatology, Eco-translatology starts with ecology, focuses on translatology by the perspective of ecology, re-explains translation phenomenon by using the research achievements of ecology, underlines the importance of general research by systematic point of view, and meanwhile attaches importance to the researches of multidimensional level and structure on the whole. Based on the basic theory of Eco-translatology, the translation methods can be summarized as multi-dimension adaption and adaptive selection, which requires the focus on entirety and relation, dynamic condition and balance, embodying ecological aesthetics in the process of translation and caring for "translation community", diversity and unification.

The perspective of Ecology leads translation to a wider academic vision. As a research model that the idea of ecology overviews the translation activities, Eco-translatology is the general research for translation from the perspective of ecology and it possesses the unique viewing angle for many key issues in translating activities. In Eco-translatology, entirety and relation are emphasized, and the individual dependence on the environment and the generating relation between individual and entirety are attached more importance. Now the Ecotranslatology has come into the second decade of development, and the research on the theory from domestic experts and scholars has deepened increasingly and the research scope has extended increasingly, so more and more academic papers have published abroad. By using Eco-translatology translation phenomena have been uncovered preferably and the problems in translation research have been solved, which was accepted and testified by experts and scholars.

In recent years from the perspective of Eco-translatology, there are only a few types of research on the translation of scitech abstracts, whereas there are less researches on sci-tech abstract translation and multi-dimensional reconstruction by using Eco-translatology. On the whole multi-layered and multidimensional analysis, there are Instructional value and significance for improving sci-tech abstract translation by suing Eco-translatology to explore language and underlying factors behind the language.

\section{THE EXISTING PROBLEMS}

The domestic scholars have played efforts on the research of abstract translation from different angles in recent 30 years, which has positive effect on the improvement of abstract translation, but most of the domestic scholars made their research on different subject categories, and meanwhile most of the current researches on sci-tech abstract translation place more emphasis on wording, tense, voice, syntax, discourse, and information, etc., so the researches are simplex and fragmented [6]. And now the researches on the translation of sci-tech paper abstract mostly focus on the specific translating techniques, whereas the discussions about deeper factors behind the linguistic superficial phenomena are insufficient. On the abstract translation fewer researches can multi-dimensionally analyze the influential factors such as translators' thinking mode, educational background, hobbies and interests, translating concept, aesthetical standard, practical experience, and meanwhile most researches ignore the objective factors such as text type, readers' demand, acceptable culture, communicative channels, normative environment, etc. in translating materials. In addition, some current researches lack intensive study on the problems that are caused by different subjective and objective environments and internal and external surroundings, and ignored the fact that translations should not only convey the original information but also consider the target readers' acceptance and the accuracy of the conveyed information, rather than the translation of literal equivalence [7]. Through the analyze to the sci-tech abstract translation in domestic core periodicals at present, it is found that currently in most translations of sci-tech paper abstract the main problems are as follows:

1. Some scholars neglect the importance of English abstract and treat English abstract with a sloppy attitude, so there are many problems in their abstract translation such as irregular formats, grammatical mistakes, and verbal errors, etc.

2. Many paper authors translate the abstract briefly by translation tools. With the development of information technology, many translation software springs up constantly such as Youdao, Google, PowerWord, etc., so copy any sentence or paragraph into the translation software and click the button "automatic translation", the relevant English translation will appear. In fact, most of the translation from the software is the combination of words in Chinese order rather than the accurate English expression.

3. Apply the English tense and voice incorrectly, which leads to the deficient demonstration of the thesis statement.

4. Be dim about the exact meaning and usage of words, which results in verbal errors.

5. Some thesis authors think that the English abstract comes from the direct translation of Chinese abstract, so their translation is the identical reproduction of the Chinese abstract: words to words and sentence to sentence, and furthermore the translation applies Chinese sentence patterns completely, which can't be faithful to the original text and often renders lots of mistakes and disordered logic.

6. The deficiency of important Chinese information or too much background information in English translation occurs frequently, and furthermore, the translations are often obscure and unaesthetic, which can't reflect the aesthetic value of scitech translation.

\section{THE RELEVANT RESEARCH OF TRANSLATION STRATEGIES}

For the translation of sci-tech paper abstract, it is inappropriate to merely focus on the language itself such as wording, tense, voice, syntax, and discourse, and the deeper factors behind the language surface layer also should be taken into full consideration. It is necessary to focus on the thought characteristics of the two nations, the relevance among different ecosystems, the showing of ecological aesthetics and multi-dimensional conversion, so the quality of translation can be improved by various reconstructions such as from information, logic and lexicon, etc. 


\section{A. Information Reconstruction}

The phenomena that the information center is dim, central information and other information are often in logical fault age often appear in the translation of sci-tech paper abstract, and this is the result of different focalizing mode of the two languages in China and the west, which is the source of information reconstruction in sci-tech abstract translation. English sentences are characterized by a focus perspective, so no matter simple sentence or subordinate clause there is a center of perceptivity, and secondary parts and subordinate clauses are related to the center in a certain way. For English sentences the language representation is organized rigorously and the boundary is clear, appearing in solid geometry of spatial relationship, while for Chinese the mode of general cognitive thinking appear cavalier perspective in sentence organization, i.e. in sentences there aren't evident features of subject-predicate core, and the objective is the complete expression of meaning, In Chinese each semantic structure is arranged smoothly to express the content, and the completeness of expressing meaning is the target.

\section{B. Logical Cohesion}

The Chinese logical cohesion is paratactic and covert. Chinese is the "invisible language", which means the cohesion of paragraphs appears in the covert and unmarked features and the usage of Chinese conjunctions often, be omitted when they can be sensed. If the conjunctions must appear in the sentence, it will be unskillful. The internal logic of Chinese needs the readers' deeply thinking, while English sentence building is featured by hypo taxis, and the connotative logic relation should be shown in language, and when multiple sub-clauses appear simultaneously it will be in the form of the subjectpredicate core with many clauses. However, many sci-tech scholars can't realize the differences and form more accurate cognition, so in the subconsciousness of their English translation, the English conjunctions are omitted habitually and as mother tongue, Chinese logic is transferred into English, and thus cause the errors. So in the Chinese to English translation, the logical cohesion of the original information should be reconstructed and the information transfer in English mode should be emphasized.

\section{Lexicon}

The Chinese words and expressions are stretchy and elastic, which leads to the unfixed features in the words'splitting and integrating. The meaning of Chinese words are flexible with wider range and the boundary of words meaning should be determined by specific context, which causes the mutual subsumption in Chinese words and they supplement each other as an integral whole, while English words are relatively independent and the meaning of English words are more concrete and clearer, and furthermore, the boundary of English words' meanings are clear. So in the Chinese to English translation, the overlapped parts of words meaning in Chinese should be separated. In addition, the flexibility of the words meaning in Chinese cause the semantic repetition easily when Chinese words aggregate mutually. The semantic repetition can be accepted in Chinese, but it should be reconstructed in Chinese to English translation.

\section{CONCLUSION}

Reading abstracts is the effective way of knowing the paper content, especially with the increasingly frequent technological and cultural communication in China and the west, more and more academic periodicals require the paper authors provide English abstracts so that it's convenient for international academic communication and information processing. The translation is a cause with strong practicality, and it requires highly for translators. For translators, they not only should be equipped with the rich language knowledge but also grasping translation theories, and meanwhile, they should apply appropriate theory to multi-dimensionally analyze the influential factors of subject and object and the internal and external surroundings. The translation of sci-tech paper abstracts are far from the problems that superficial translation techniques of language can solve, and it is the coordination and game between two cultures of east and west, and it is the process of re-offering a set of solutions after the translators' insight of the differences in the two languages. Based on the features of the target language, the process is not only the reconstruction of the linguistic form but also the reconstruction of the two philosophizing and cultures, so it's necessary for the translators to well grasp the features of the relevant sci-tech language and are familiar with intellectual and cultural differences behind the two languages. Through the discussion about a sci-tech abstract translation, it is hoped to improve translation and appreciation levels of the translators and scitech workers.

\section{REFERENCES}

[1] YU Li. The Reconstruction of Appraisal Meaning in Novel Translation, Foreign Language Research, 2019, (01), 57-62. (In Chinese)

[2] XIA Yong-qiang. On science and technology English stylistic features and translation skills, Science \& Technology Vision, 2017, (22), 72-73. (In Chinese)

[3] TAN Meng, On Conceptual Reconstruction in the Process of Translating From the Perspective of Concept Types, Journal of Guangdong University of Foreign Studies, 2016, 27(01), 73-80. (In Chinese)

[4] MA Jun. Reconstitution of the Classroom Teaching of English-Chinese Cultural Translation in Colleges and Universities from the Perspective of Cohesion Theory, Journal of Inner Mongolia University of Finance and Economics, 2019, 17(03), 111-113. (In Chinese)

[5] WEN Bin-bin, CHANG Chun. Translation of terms in thesauri based on eco-translatology, Chinese Journal of Medical Library and Information Science, 2019, 28(02), 50-54. (In Chinese)

[6] LI Li-jun, GUO Man-rui. Empirical Study on the Translation Teaching Mode of College English from the Perspective of Eco-translatology, Journal of North China Electric Power University, 2018, (06), 123-129. (In Chinese)

[7] DONG Lina. Chinese Translation of Popular Science Writing in the Perspective of Eco-translatology, Journal of Tongren University, 2018, 20(10), 79-82+128. (In Chinese) 